\title{
Studies of fission fragment yields via high-resolution $\gamma$-ray spectroscopy
}

J.N. Wilson ${ }^{1}$, M. Lebois ${ }^{1}$, L. Qi ${ }^{1}$, P. Amador-Celdran ${ }^{2}$, D. Bleuel ${ }^{3}$, J.A. Briz ${ }^{4}$, R. Carroll ${ }^{5}, W$. Catford $^{5}$, H. De Witte ${ }^{6}$, D.T. Doherty ${ }^{7}$, R. Eloirdi ${ }^{2}$, G. Georgiev ${ }^{8}$, A. Gottardo ${ }^{1}$, A. Goasduff ${ }^{8}$, K. Hadyñska-Klek ${ }^{9}, K$. Hauschild ${ }^{8}, H$. Hess $^{10}$, V. Ingeberg ${ }^{11}$, T. Konstantinopoulos ${ }^{8}$, J. Ljungvall ${ }^{8}$, A. Lopez-Martens ${ }^{8}$, G. Lorusso ${ }^{12}, R$. Lozeva ${ }^{8}, R$. Lutter $^{13}$, P. Marini ${ }^{14}, I$. Matea ${ }^{1}$, T. Materna ${ }^{7}$, L. Mathieu ${ }^{15}$, A. Oberstedt ${ }^{16}, S$. Oberstedt ${ }^{17}$, S. Panebianco ${ }^{7}$, Zs. Podolyak ${ }^{5}$, A. Porta ${ }^{4}$, P.H.

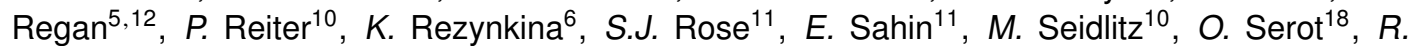
Shearman $^{512}$, B. Siebeck ${ }^{10}$, S. Siem ${ }^{11}$, A.G. Smith ${ }^{19}$, G.M. Tveten ${ }^{11}$, D. Verney ${ }^{1}$, N. Warr ${ }^{10}$, F. Zeiser ${ }^{11}$, and $M$. Zielinska ${ }^{7}$

${ }^{1}$ Institut de Physique Nucléaire, CNRS-IN2P3, Univ. Paris-Sud, Université Paris-Saclay, 91406 Orsay

Cedex, France

${ }^{2}$ European Commission, Joint Research Centre, Directorate G for Nuclear Safety and Security,Unit G.I.5, Postfach 2340, 76215 Karlsruhe, Germany

${ }^{3}$ Lawrence Livermore National Laboratory, 7000 East Ave, Livermore, CA 94550, USA

${ }^{4}$ Subatech/Ecole des Mines, 4 rue Alfred Kastler, 44307 Nantes, France

${ }^{5}$ Department of Physics, University of Surrey, Guildford, GU2 7XH, UK

${ }^{6}$ Institute for Nuclear and Radiation Physics, KU Leuven, 3000 Leuven, Belgium

${ }^{7}$ Irfu, CEA, Université Paris Saclay, 91191 Gif-sur-Yvette Cedex, France

${ }^{8}$ CSNSM Orsay, CNRS-IN2P3, Univ. Paris-Sud, Université Paris-Saclay, 91405 Orsay Cedex, France

${ }^{9}$ INFN Laboratori Nazionali di Legnaro, Viale dell'Universita, 2, 35020 Legnaro, Italy

${ }^{10}$ Institut für Kernphysik, Zülpicher Strasse 77, 50937 Köln, Germany

${ }^{11}$ University of Oslo, Department of Physics, P.O. Box 1048, Blindern 0316 Oslo, Norway

${ }^{12}$ National Physical Laboratory, Hampton Road, Teddington, TW11 OLW, UK

${ }^{13}$ LMU München, Schellingstrasse 4, 80799, Munich, Germany

${ }^{14}$ CEA/DAM, DIF, Bruyères-le-châtel, 91297 Arpajon, France

${ }^{15}$ CENBG Bordeaux, Chemin du Solarium, Le Haut Vigneau, BP. 120, 33175 Gradignan, France

${ }^{16}$ Extreme Light Infrastructure - Nuclear Physics (ELI-NP), 077125 Bucharest-Magurele, Romania

${ }^{17}$ European Commission, Joint Research Centre, Directorate G for Nuclear Safety and Security, Unit G.2, 2440 Geel, Belgium

${ }^{18}$ CEA Cadarache, 13108 Saint-Paul-lez-Durance, France

${ }^{19}$ University of Manchester, Dept. Of Physics, Oxford Road, Manchester, M13 9PL, UK

\footnotetext{
Abstract. Precise spectroscopic information on the fast neutron induced fission of the ${ }^{238} \mathrm{U}(\mathrm{n}, \mathrm{f})$ reaction was recently gained using a new technique which involved coupling of the Miniball high resolution $\gamma$-ray spectrometer and the LICORNE directional neutron source. The experiment allowed measurement of the isotopic fission yields for around 40 even-even nuclei at an incident neutron energy of around $2 \mathrm{MeV}$ where yield data are very sparse. In addition spectroscopic information on very neutron-rich fission products was obtained. Results were compared to models, both the JEFF-3.1.1 data base and the GEF code, and large discrepancies for the S1 fission mode in the Sn/Mo isotope pair
} 
were discovered. This suggests that current models are overestimating the role played by spherical shell effects in fast neutron induced fission. In late 2017 and 2018 the nu-ball hybrid spectrometer will be constructed at the IPN Orsay to perform further experimental investigations with directional neutrons coupled to a powerful hybrid $\mathrm{Ge} / \mathrm{LaBr} 3$ detector array. This will open up new possibilities for measurements of fission yields for fastneutron-induced fission using the spectroscopic technique and will be complimentary to other methods being developed.

\section{Introduction}

The measurement of the isotopic distribution of fission fragments or fission yields and how they vary as a function of the mass, charge, excitation energy and angular momentum of the fissioning system is very important for a deeper understanding of nuclear fission. The precise details of the mass and charge splits are determined by the complex shell effects in the nascent fragments and parent nucleus. Fission models must be capable of reproducing these fragment distributions with a high degree of accuracy. Furthermore, for neutron induced fission in particular the knowledge of fission yields has some important consequences for energy applications and the functioning of reactors. These are the production of neutron poisons (e.g. ${ }^{135} \mathrm{Xe}$ ), the production of beta-delayed neutron emitters (e.g. ${ }^{137} \mathrm{I}$ ), the decay heat, the production of long-lived waste components (e.g. ${ }^{137} \mathrm{Cs}$ ) and the emitted reactor anti-neutrino flux and spectra. This latter point is important for both non-invasive core monitoring and anti-proliferation and short base-line anti-neutrino experiments and short range neutrino oscillations.

There are several methods of obtaining experimental information on fission yields that have been developed over the last few decades. Simple mass yield curves can be obtained via radiochemical separation and spectroscopy after an actinide sample is irradiated with neutrons [1]. However, obtaining the fully characterised yields in mass and charge of the fragments is much more experimentally challenging. For thermal neutron induced fission extensive measurements have been performed over the last two decades with recoil mass spectrometers such as Lohengrin [2]. For fast neutron induced fission yield measurements exist for $14 \mathrm{MeV}$ neutrons thanks to the very high neutron fluxes possible from neutron generators using the $t(d, n)^{4} \mathrm{He}$ reaction. However, for energies in between these two extremes which are most relevant for fast reactor applications $(1-4 \mathrm{MeV})$ the current experimental data are very sparse.

\subsection{Surrogate reaction yield measurements}

Two recent techniques have been able to provide complete fission yield data in this intermediate energy region using surrogate reactions in inverse kinematics. This requires acceleration of actinide nuclei to hundreds of MeV per nucleon. The SOFIA experiment [3] at GSI uses photofission as the reaction mechanism which achieves complete characterisation of both fission fragments and the emitted neutrons on an event-by-event basis. Fragment distributions for fissioning nuclei over a wide range of $\mathrm{A}$ and $\mathrm{Z}$ can be measured leading to a wealth of new and important experimental information. However, the experiment is currently unable to control for compound nucleus excitation energy, which is on average high $(11 \mathrm{MeV})$ and has a broad distribution. This makes direct comparison with fastneutron-induced fission difficult.

The Vamos/Spider [4] setup at GANIL uses multi-nucleon transfer reactions in inverse kinematics to induce fission. The main advantage is that the detection of the outgoing ejectiles from the transfer reaction in the spider charged particle detector allows for reconstruction of the compound nucleus excitation energy if no neutron emission is assumed. However, only one of the two fragments is 
Theory-4

measured in the VAMOS mass spectrometer and the multi-nucleon transfer reaction can provide a large angular momentum transfer to the compound system (beyond $20 \mathrm{hbar}$ ). The angular momentum distribution of the fissioning system is thus radically different from that of the equivalent neutroninduced reaction for similar excitation energies. It is not at all clear what effect such differences would have on resultant isotopic yields. However, nuclear shell effects are known to change as a function of spin, and thus it would be surprising if large differences in initial angular momenta would not have some effect on the resulting yield distributions.

\section{$1.22 \mathrm{E}-2 \mathrm{v}$ measurements}

The most direct and potentially accurate method to perform yield measurements is using a double armed $2 \mathrm{E}-2 \mathrm{v}$ spectrometer which directly measures the kinetic energy of each fragment, and the precision time-of-flight (TOF) over the flight path for each arm. This gives access to pre and post neutron masses, and charges through energy loss measurements. At least four of these devices are under development (e.g. STEFF [5], SPIDER [6], FALSTAFF [7] and VERDI [8]). Eventually the devices will be used with fast neutron beams available at new facilities such as NFS. However, the actinide samples used must be extremely thin to avoid energy losses and hence limited to less than $1 \mathrm{mg}$ of material. In the best case fission rates of a few per second seems feasible at the highest neutron fluxes. However, the intrinsic efficiencies for detecting fission fragments is very low (typically 1-3\%) due to the small solid angle coverage of the detectors in the spectrometer arms. This implies very long experiments will be needed to gather sufficient statistics, even with state-of-the-art intense neutron beams from new high-flux facilities.

\subsection{Prompt spectroscopic methods}

One rarely-used method for measuring fission yields, whose potential has possibly been overlooked, is the use of high-resolution gamma-ray spectroscopy to measure the prompt gamma rays de-exciting the fission fragments. Each fragment has a unique gamma decay signature dependent on its particular nuclear structure and the pathways via which its excited states decay. To uniquely identify fragments requires double or triple gamma ray coincidence spectroscopy, which in turn needs a high efficiency, high resolution gamma ray spectrometer using Germanium detectors.

The spectroscopic method was first used to measure yields of a ${ }^{252} \mathrm{Cf}$ spontaneous source [9] at Oak ridge in the 1990's with an array of 12 Ge detectors. The method was subsequently deployed to measure fission yields in the heavy ion induced ${ }^{208} \mathrm{~Pb}\left({ }^{18} \mathrm{O}\right.$,f) reaction with the Euroball spectrometer [10]. However, until recently this method has been essentially limited to yield measurements of spontaneous fission since the technique has particular problems correcting for isomers (underproduction) and fragment population via beta-decay (overproduction).

However, with the recent coupling of the MINIBALL spectrometer [11] to the LICORNE directional neutron source $[12,13]$, this method can now be extended to direct measurements of yields for fast-neutron-induced fission.

The spectroscopic method has certain limitations so it currently cannot achieve the same level of precision as other techniques. However, relative errors of less than $20 \%$ seem feasible. The measurement precision is limited by at least four factors:

(i) The detection efficiency of the spectrometer must be known as accurately as possible. At the lowest gamma ray energies $(<200 \mathrm{keV})$ this can prove difficult, and introduce systematic errors to the measurement. 
(ii) A sum of all the gamma intensity feeding the first excited states must be carried out to determine the absolute yield. This can be particularly challenging for odd nuclei where the intensity is fragmented and there are many weakly populated decay paths feeding the first excited state.

(iii) Since the technique is dependent on gamma coincidence measurements it is impossible to determine the proportion of total intensity directly feeding the ground state for any particular fragment. Although this is expected to be quite small in most cases, the ground state feeding may vary between nuclei and hence introduce some small systematic errors.

(iv) Large corrections may be necessary for missing intensity due to the presence of isomeric states or beta decay.

In our experiments we have shown that the latter point can be mitigated by use of a pulsed beam which exploits time correlations to allow measurement of missing intensity from isomeric states and simultaneously strongly selects against uncorrelated beta-decays.

\section{Experimental details}

In February 2015, the LICORNE directional neutron source was successfully coupled to the Miniball spectrometer at the IPN Orsay. The full details of the LICORNE/MINIBALL experiment can be found here [14]. LICORNE is an inverse kinematic neutron source based on the $\mathrm{p}\left({ }^{7} \mathrm{Li}, \mathrm{n}\right)^{7} \mathrm{Be}$ reaction which can achieve high fluxes of up to $10^{7} \mathrm{n} / \mathrm{s} / \mathrm{cm}^{2}$ for samples placed a few $\mathrm{cm}$ away from the source.

The MINIBALL spectrometer consists of 24 Ge crystals packaged in 8 triple-clusters. Each cluster was placed approximately $14 \mathrm{~cm}$ from the fission target, and the array covered one third of the total solid angle, providing a total photopeak efficiency of around $6 \%$ at $1 \mathrm{MeV}$. The LICORNE directional neutrons had an average energy of $1.7 \mathrm{MeV}$ just above the ${ }^{238} \mathrm{U}$ fission threshold. The maximum neutron cone angle was 19.5 degrees and the beam was pulsed with a period of $400 \mathrm{~ns}$ and pulse width of $2 \mathrm{~ns}$. A sample of $31.35 \mathrm{~g}$ of depleted ${ }^{238} \mathrm{U}$ was irradiated over an effective beam time of 11 days with fissions induced at an estimated rate of $25 \mathrm{kHz}$.

The MINIBALL array was designed as a high-efficiency, low-multiplicity device for radioactive beam experiments and is not Compton suppressed. The peak-to-total is fairly low $22 \%$, and thus limits the resolving power of the device when used to select nuclei using standard double or triple gamma coincidence techniques. The Compton background in MINIBALL is particularly large at energies in the $200-500 \mathrm{keV}$ range. Furthermore, self-shielding of low energy gamma rays by the compact metal target limited the sensitivity of the experiment below $500 \mathrm{keV}$. Measurements of yields for odd nuclei were thus particularly challenging and hence the published results focused on the 40 most strongly populated even-even nuclei in the fission of ${ }^{238} \mathrm{U}$.

\section{Results}

The absolute yields measured in the experiment can again be found in our recent publication [14], but are seen to be reasonably well-reproduced by the JEFF3.3.1 and ENDFB7 evaluations and the GEF fission code apart from the yields of $\mathrm{Sn} / \mathrm{Mo}$ isotopes which showed significant deviations. This is interpreted as a quenching of the S1 fission mode and an overestimation of the role of spherical shell effects in fission for this particular nucleus.

A gamma ray coincidence spectrum of a typical gate on the $2^{+} \rightarrow 0^{+}$transition in a strongly populated even-even fragment can be seen in Fig. 1. The spectrum shows both the higher-lying transitions in ${ }^{96} \mathrm{Sr}$ and also low-lying transitions from all its binary partners ${ }^{142} \mathrm{Xe},{ }^{141} \mathrm{Xn},{ }^{140} \mathrm{Xe}$, ${ }^{139} \mathrm{Xe}$, etc. corresponding to evaporation of $1,2,3,4$ etc. neutrons. 


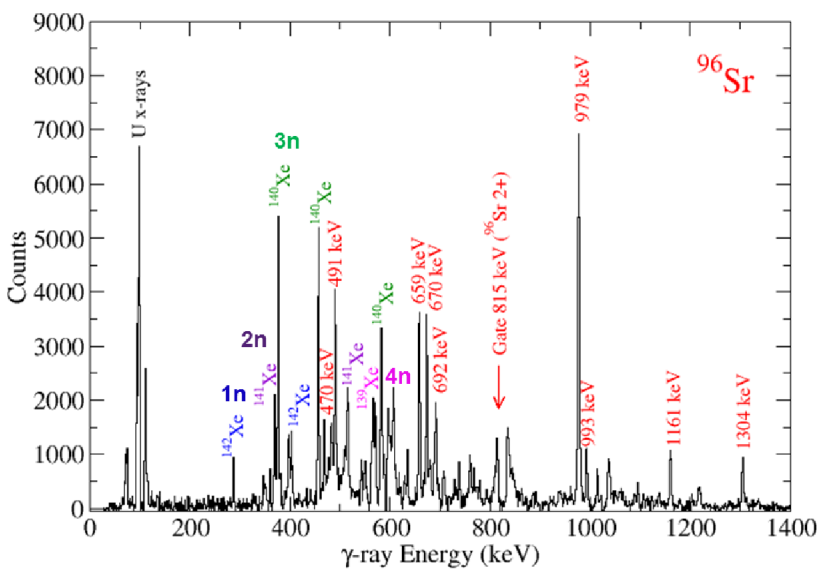

Figure 1. A typical gamma ray coincidence spectrum obtained from gating on the $2^{+} \rightarrow 0^{+}$transition in ${ }^{96} \mathrm{Sr}$ at $815 \mathrm{keV}$. Coincidences from gamma rays emitted from higher lying states in ${ }^{96} \mathrm{Sr}$ can be observed along with the corresponding binary partners of ${ }^{142-139} \mathrm{Xe}$.

An interesting possibility for a more complete analysis of the gamma-ray coincidence data, arises from the collection of correlated fragment information. It is possible to deduce the relative probabilities for the number of neutrons emitted conditional on the detection of ${ }^{96} \mathrm{Sr}$ by measuring the relative intensities of its binary Xe partners. If combined with similar data from all the other $\mathrm{Sr}$ fragments populated in the experiment, then a neutron multiplicity distribution can be deduced for the $\mathrm{Sr} / \mathrm{Xe}$ charge split.

It has been relatively straight forward to extract this information from the LICORNE/MINIBALL data for $\mathrm{Sr}$ and Xe because the first excited states of both odd and even $\mathrm{Sr}$ and Xe nuclei are relatively high in energy, hence intensity measurements didn't suffer from the sample self-shielding problem which normally occurs for odd nuclei with low energy gamma transitions. Figure 2 shows graphically the summary of these absolute yield measurements for the observed Xe partners correlated with every observed Sr isotope. (It should be noted that the measurement could be performed again independently by gating on all observed Xe isotopes and measuring the corresponding $\mathrm{Sr}$ binary partners).

The first observation from this figure is that the more stable the selected $\mathrm{Sr}$ isotope, the more neutrons on average are evaporated and vice-versa. For example, the $8 \mathrm{n}$ neutron channel becomes just visible when gating on the ${ }^{94} \mathrm{Sr}$ closest to stability, and the $2 \mathrm{n}$ and $3 \mathrm{n}$ channels dominate when selecting the most exotic Sr isotopes. This correlation is clearly as it ought to be. The second observation is that the distribution of emitted neutrons appears to become broader when moving closer to stability. This also appears reasonable since there are many more available combinations for the number of neutrons emitted by each $\mathrm{Sr}$ and $\mathrm{Xe}$ fragment when the sum is larger on average.

Once the complete correlated yield matrix has been extracted for the particular charge split (in this case $\mathrm{Se} / \mathrm{Xe}$ ) it is possible to sum the results for each neutron multiplicity. Hence the complete neutron multiplicity distribution for the isotope pair can be deduced. This can be seen in Fig. 3 .

\subsection{The nu-ball array and improvements to the spectroscopic technique}

In 2017 and 2018 an experimental campaign with the nu-ball spectrometer is planned. Nu-ball consists of $34 \mathrm{Ge}$ detectors 24 clovers and 10 coaxial coupled to $20 \mathrm{LaBr} 3$ detectors from the FATIMA 


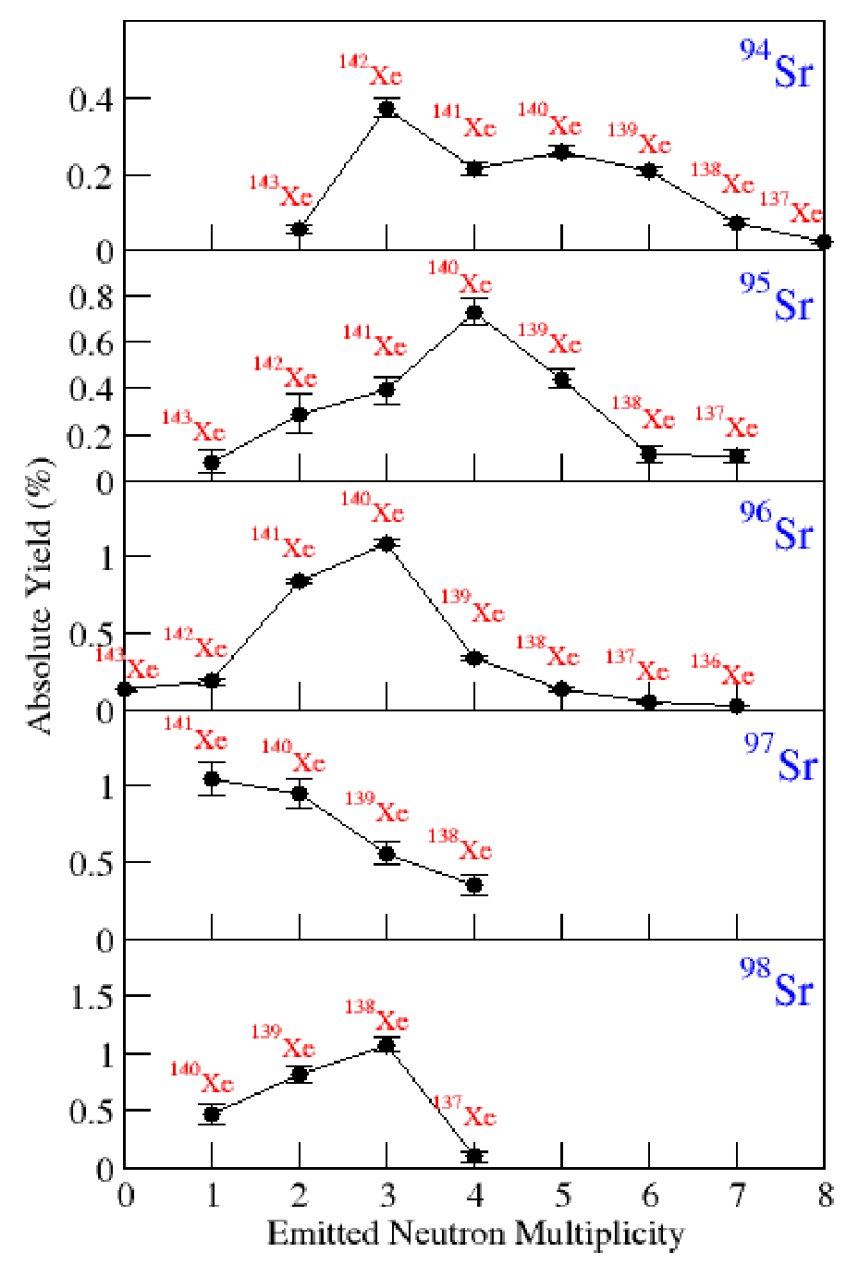

Figure 2. Absolute yields of correlated Sr-Xe nuclide pairs.

collaboration. It is currently under construction at the IPN Orsay and will be commissioned in October 2017. The photopeak efficiency at $1 \mathrm{MeV}$ is expected to be 5.5\%. Nu-ball aims to improve on the resolving power of MINIBALL by at least an order of magnitude since the Ge detectors have antiCompton BGO shields and will be Compton suppressed. This will raise the peak-to-total ratio of the device at $1 \mathrm{MeV}$ from $20 \%$ to around 50\%. In addition, the self-shielding of the massive actinide samples to be irradiated with neutrons at the centre of the spectrometer (tens of grams of ${ }^{238} \mathrm{U}$ and ${ }^{232} \mathrm{Th}$ ) will be hugely diminished. The newly developed metal samples will have average densities of between 0.5 and $1.0 \mathrm{~g} / \mathrm{cm}^{3}$ compared to a density of $19.1 \mathrm{~g} / \mathrm{cm}^{3}$ for uranium metal. The reduction in effective thickness of material that gamma rays must travel through to escape the surface can be shown to be proportional to a power of the ratio of densities $\left(\rho^{\text {new }} / \rho^{\text {old }}\right)^{2 / 3}$.

Therefore, reducing the density of the sample by a factor of 40 in our case will produce a corresponding reduction in the gamma-ray attenuation of the sample by over a factor of 10 and hugely increase the efficiency of the spectrometer in the low energy range (100-500 keV). Lowering the 


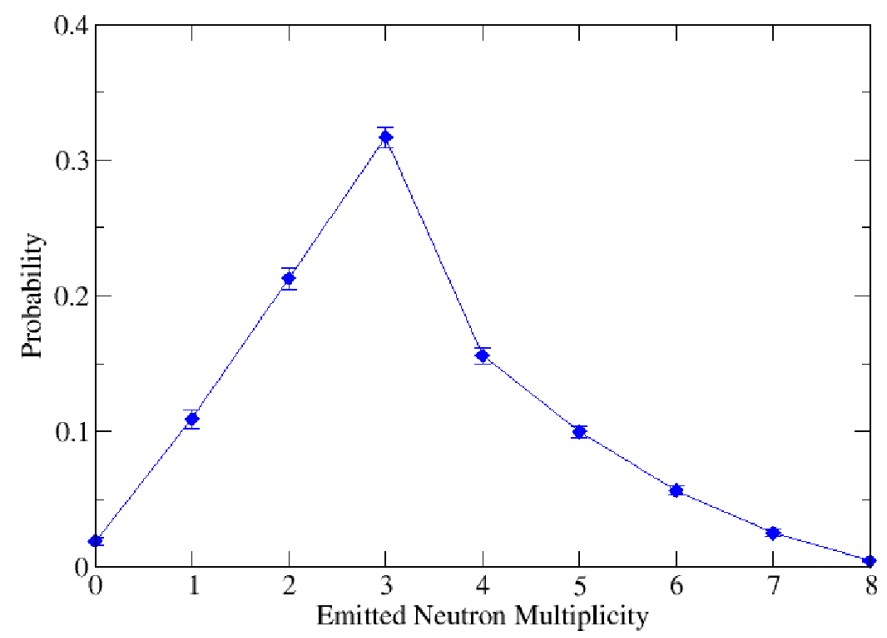

Figure 3. Deduced neutron multiplicity distribution for the $\mathrm{Sr}-\mathrm{Xe}$ isotope charge split.

average density of the actinide samples is achieved by extending the sample in space over several centimetres, either by stacking spaced out thin foils, or by encapsulating metallic turnings.

\section{Conclusion}

The combination of a fast directional, neutron source coupled to a high-resolution gamma-ray spectrometer has been used to demonstrate the feasibility of making direct fission-yield measurements for fast-neutron induced fission in $1-4 \mathrm{MeV}$ range. This energy range is very important for energy applications for both current and future (Gen IV) nuclear reactors yet current yield data are very sparse.

The recent LICORNE/MINIBALL experiment has also shown the importance of a pulsed neutron beam for accurate yield measurements with gamma spectroscopy which is essential to suppress beta decay and correct for the presence of isomers. Furthermore, the spectroscopic technique produces correlated fragment data which allows extraction of neutron multiplicity distributions for different fragment charge splits. This is an additional observable in fission that can now be compared with theory and hence may allow for further refinement of fission models and a deeper understanding of the fission process.

Improvements are currently being made to the spectroscopic technique to measure fragment yields for fast-neutron induced fission. The nu-ball spectrometer is currently under construction at the IPN Orsay which will improve the resolving power by an order of magnitude and samples with a much lower average density are being designed to solve the self-shielding problem. These improvements should allow higher precision yield measurements of ${ }^{238} \mathrm{U}(\mathrm{n}, \mathrm{f})$ and ${ }^{232} \mathrm{Th}(\mathrm{n}, \mathrm{f})$ in the near future. The samples to be used in these cases will contain tens of grams of actinide material. However, with the improved resolving power of the spectrometer and the potential coupling to active (ionization chamber) targets, it ought to be possible to perform yield measurements with only hundreds of $\mathrm{mg}$ of material and lower fission rates of the order of $\sim 1 \mathrm{kHz}$. Hence yield measurements for the key fissile isotopes of ${ }^{235} \mathrm{U},{ }^{233} \mathrm{U}$ and ${ }^{239} \mathrm{Pu},{ }^{241} \mathrm{Pu}$ may soon be within reach with the gamma spectroscopic technique for fast-neutron-induced fission. These will hopefully provide a useful compliment to measurements with the different $2 \mathrm{E}-2 \mathrm{v}$ techniques planned for future fast-neutron facilities such as NFS. 


\section{References}

[1] S. Nagy, K.F. Flynn, J.E. Gindler, J.W. Meadows, and L.E. Glendenin, Phys. Rev. C 17, 163 (1978)

[2] H.G. Börner and F. Gönnenwein, The Neutron: A Tool and an Object in Nuclear and Particle Physics, World Scientific, Singapore (2012)

[3] L. Grente et al., EPJ Web of Conferences 122, 01006 (2016)

[4] F. Farget et al., Eur. Phys. J. A 51, 175 (2015)

[5] A.J. Pollitt, A.G. Smith, I. Tsekhanovich, J.A. Dare and L. Murray, EPJ Web of Conferences 93, 02018 (2015)

[6] F. Tovesson, et al., EPJ Web of Conferences 62, 05002 (2013)

[7] D. Doré et al., Nuclear Data Sheets 119, 346-348 (2014)

[8] M.O. Frégeau et al., EPJ Web of Conferences 62, 05003 (2013)

[9] G.M. Ter-Akopian et al., Phys. Rev. C 55, 3 (1997)

[10] A. Bogachev et al., Eur. Phys. J. A 34, 23-28 (2007)

[11] N. Warr et al., Eur. Phys. J. A 49, 40 (2013)

[12] M. Lebois, J.N. Wilson, P. Halipré, B. Leniau, I. Matea, A. Oberstedt, S. Oberstedt, and D. Verney, Nucl. Instrum. Methods Phys. Res., Sect. A 735, 145 (2014)

[13] J.N. Wilson, M. Lebois, P. Halipré, S. Oberstedt, and A. Oberstedt, Phys. Procedia 64, 107 (2015)

[14] J.N. Wilson et al., Phys. Rev. Lett. 118, 222501 (2017) 